\title{
Análisis isocinético de rodilla en adultos mayores con gonartrosis grado I-II
}

\author{
Arriaga-Rivera Javier*, Alejo-González Mónica Patricia**, García-Pérez Angélica \\ Elizabeth ${ }^{* * *}$,Landeros-Gallardo Carlos**, Pech-Moguel Gladys Antonia***
}

\begin{tabular}{|c|c|}
\hline 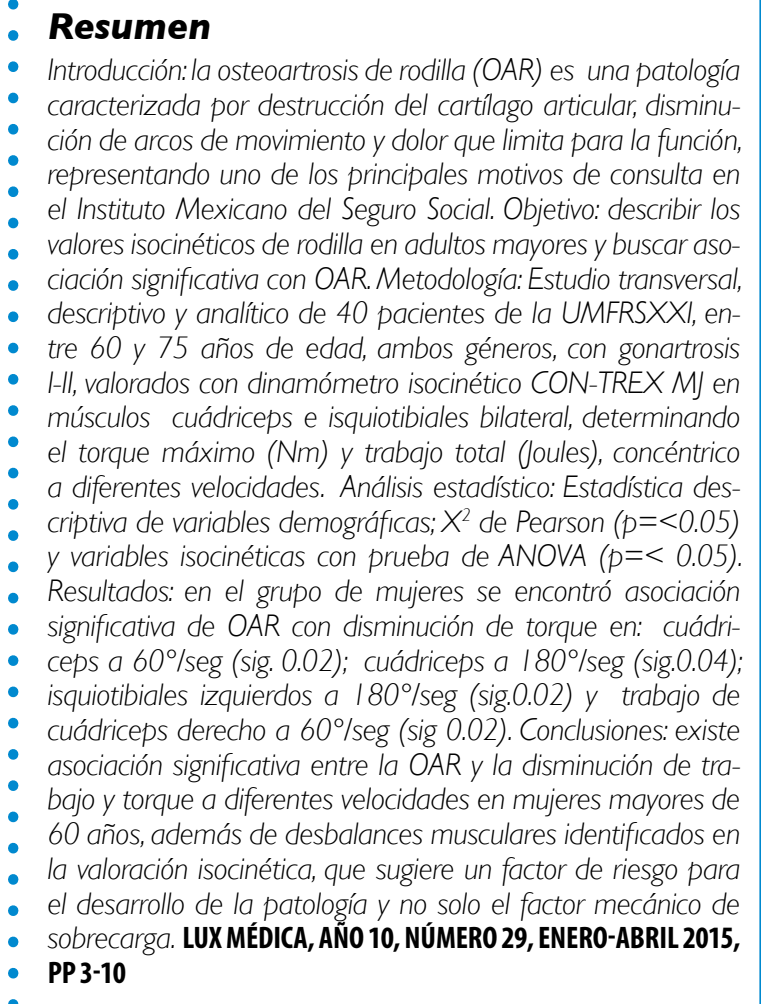 & 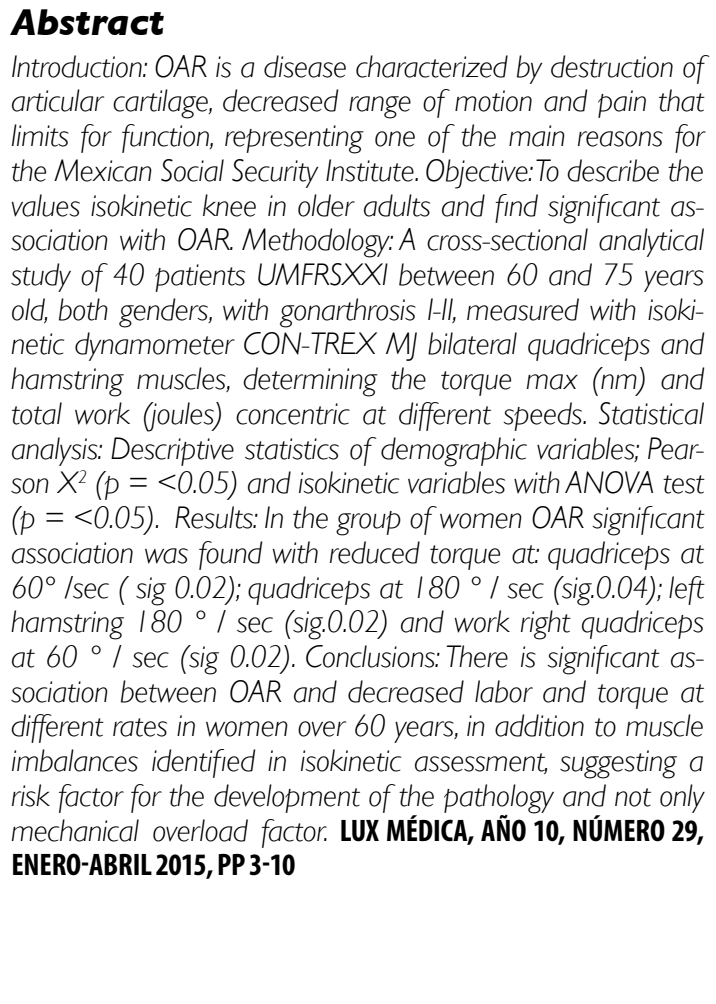 \\
\hline $\begin{array}{r}\text { Palabras clave: cuádriceps, isocinética, trabajo, tor } \\
\text { adulto mayor, osteoart }\end{array}$ & $\begin{array}{l}\text { Iords: quadriceps isokinetic, work, torque, elderly, } \\
\text { arthritis }\end{array}$ \\
\hline & \\
\hline
\end{tabular}

* Médico especialista en Medicina de Rehabilitación. Subdirector médico de clínica del CRIT Guerrero.

** Médico especialista en Medicina de Rehabilitación y Alta especialidad en Electrodiagnóstico. Adscrita al servicio de electrodiagnóstico en el CRIT Guerrero.

*** Médico especialista en Medicina de Rehabilitación. Adscrita a la Unidad de Medicina Física y Rehabilitación siglo XXI.

Fecha de recibido: 28 de enero 2015

Fecha de aceptación: 28 de febrero 2015

Correspondencia: Dr Javier Arriaga Rivera. Blvd. de las Naciones No. 2607 Colonia Antigua Hacienda El Potrero. Código Postal 39906 Acapulco, Guerrero, México. Teléfono +01 (744) 4341500 Correo electrónico: arriaga@teleton-gro.org.mx 


\section{Introducción}

La osteoartrosis de rodilla (OAR) es una enfermedad articular caracterizada por degeneración, pérdida del cartílago y alteración del hueso subcondral, asociada a cambios en tejidos blandos. El diagnóstico es clínico. ${ }^{1,2,3}$ Sin embargo en ocasiones el control de las secuelas del mismo se prolonga con dolor crónico, deformidad, luxaciones y finalmente requiere de métodos invasivos como la colocación de prótesis en medida extrema de tratamiento. ${ }^{1}$ En México se estima que la prevalencia de OA es de $10.5 \%$, más frecuente en mujeres $(11.7 \%)$ que en hombres $(8.71 \%)$, con variaciones importantes en las distintas regiones del país: en Chihuahua $20.5 \%$, Nuevo León $16.3 \%$, Distrito Federal $12.8 \% .^{2}$ El $15.55 \%$ de los derechohabientes del Instituto Mexicano del Seguro Social (IMSS) tienen más de 65 años de edad. Ellos demandan el 25\% de consultas de medicina familiar, el $23 \%$ de hospitalizaciones y $20 \%$ de las prestaciones sociales. En el IMSS la OAR se encuentra dentro de las 10 primero motivos de consulta en primer nivel de atención. ${ }^{4}$

La debilidad de los músculos cuádriceps ha sido correlacionada con la presencia de gonalgia en los pacientes con OAR. Esta debilidad en la musculatura tanto de flexores como extensores de rodilla, disminuye la estabilidad de la articulación y la capacidad de atenuación de las cargas. ${ }^{5}$ La dinamometría isocinética utiliza la tecnología informática y robótica para obtener y procesar la capacidad muscular en datos cuantitativos, a velocidad constante. Es un sistema objetivo de evaluación de la fuerza muscular en movimiento. La evaluación por dinamometría isocinética es usada en las últimas décadas como método para determinar el patrón funcional de fuerza y equilibrio muscular a través de las variables isocinéticas "pico de torque" y "relación de equilibrio agonista/antagonista. ${ }^{6}$

La utilización de los resultados de una evaluación isocinética posibilita una nueva herramienta y estrategia para la restructuración del plano de entrenamiento, pues permite una sensible identificación de la función muscular.? Además, se pueden determinar valores de referencia para la función muscular, en especial prediciendo el riesgo de lesiones. ${ }^{8}$ La disminución del torque en la OAR es multifactorial, generada por cambios patológicos en la marcha, pérdida de fibras musculares y sus propiedades contráctiles, así como patrones de activación muscular alterados, aun en casos clínicamente sin relevancia. El torque en músculos de rodilla de pacientes con osteoartrosis puede presentar una reducción del $20 \%$ al $40 \%$ en comparación con personas jóvenes sanas. ${ }^{9}$

El complemento diagnóstico de OAR, con el método isocinético, nos permite evaluar la funcionalidad articular y muscular de la rodilla cuantitativa y objetivamente, permitiendo identificar alteraciones musculares y establecer un programa de rehabilitación más eficiente. En México no existen estudios de valores isocinéticos en rodilla para adultos mayores, por lo que resulta importante 
tener valores de referencia propios con la finalidad de comprender mejor el comportamiento de la patología. El objetivo de este estudio es describir los valores isocinéticos, variables demográficas y determinar asociación entre las variables isocinéticas y OAR en la población de adultos mayores de la Unidad de Medicina Física y Rehabilitación Región Sur, Siglo XXI del IMSS.

| | | | | | | | | | | | | | | | | | | | | | | | | | | | | | | | | | | | | | | | | | | | | | | | | | | | | | | | | | | | | | | | | | | | | | | | | | | | | | | | | | | | | | | | | | | | | | | | | | | | | | | | | | | | | | |

\section{Material y métodos}

Estudio clínico, transversal, descriptivo y analítico, realizado en el laboratorio de Isocinecia de la Unidad de Medicina Física y Rehabilitación región sur Siglo XXI del Instituto Mexicano del Seguro Social, por muestreo no probabilístico que se obtuvo de los pacientes adultos mayores derechohabientes. El universo de estudio fue de 60 pacientes de ambos géneros con edad de 60 a 75 años que cumplieran con los siguientes criterios: con gonartrosis grado I y II en base a la clasificación radiológica de Kellgren y Lawrence, sin limitación de los arcos de movilidad y dolor articular. Se excluyeron a pacientes con antecedentes de cirugía articular de rodilla, fracturas en miembros pélvicos, lesiones de nervio periférico en miembros inferiores, dolor durante la prueba isocinética y pacientes con lesiones musculares que presentaron dolor durante la prueba. Se eliminaron a pacientes que no concluyeron la prueba isocinética. 40 pacientes cumplieron con los criterios de selección (20 hombres y 20 mujeres). Posteriormente se realizó una valoración isocinética en el equipo CONTREX MJ con los siguientes parámetros; paciente sentado con cadera, rodilla y tobillo a $90^{\circ}$, sujetado por cintillas estabilizadoras, el eje de rotación del brazo del dinamómetro se colocó lateral al epicóndilo femoral a $0^{\circ}$ de rotación y con una inclinación del asiento a $85^{\circ}$, en modalidad isocinético clásico, concéntrico/concéntrico para ambas rodillas a velocidades de $60^{\circ} / \mathrm{seg}$ (5 repeticiones), $180^{\circ} / \mathrm{seg}$ (10 re- peticiones) y $300 \%$ seg (15 repeticiones), para cuádriceps e isquiotibiales, con un periodo de descanso entre cada prueba de 60 segundos y tomando como valores de estudio: torque y trabajo total. Antes de cada prueba se le permitió al paciente realizar 5 repeticiones a $300^{\circ} / \mathrm{seg}$ para familiarizarse con el equipo (véase figura 1).

El análisis de los resultados se realizó mediante paquete estadístico SPSS ver.13,

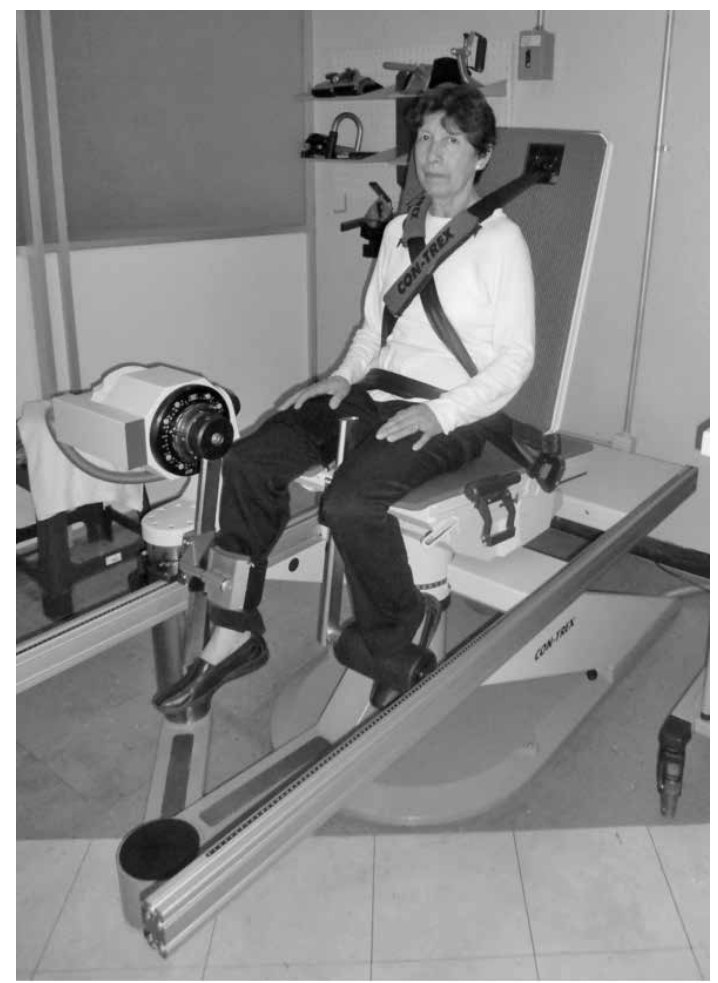

Figura 1. Muestra de posicionamiento del paciente en equipo CON-TREX MJ 
aplicando las prueba de ANOVA para análisis de variables isocinéticas y grado de OAR $(p=<0.05)$, así como $X^{2}$ de Pearson para las variables demográficas $(p=<0.05)$. Todos los pacientes fueron informados previamente sobre el estudio $y$ consintieron por escrito a participar en él; este estudio fue aceptado por el Comité de Ética e investigación local, se realizó bajo las normas éticas institucionales y acuerdos de la $18^{\circ}$ Asamblea Mundial de Helsinki.

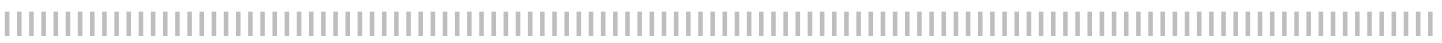

\section{Resultados}

El promedio de edad para la población derechohabiente de la Unidad de Medicina Física y Rehabilitación Siglo XXI fue: para hombres: 67.5 años y en mujeres: 65.5 años. La distribución de OAR por género fue la siguiente; en hombres el $20 \%$ presentó gonartrosis grado I y el $80 \%$ restante gonartrosis grado II. En mujeres el 30\% presentó gonartrosis grado I y el $70 \%$ gonartrosis grado II. No se encontró asociación significativa de mayor grado de OAR $y$ género $(p>0.05)$. El índice de masa corporal (IMC) en general fue: peso normal o ideal $32.5 \%$, sobrepeso $47.5 \%$, obesidad $20 \%$. No se encontró asociación entre género e IMC con la prueba de $\mathrm{X}^{2}$ de Pearson $(p>0.05)$.

Los resultados obtenidos para los valores promedio de torque en Newtons metro (Nm) y trabajo total expresado en Joules para los músculos de cuádriceps e isquiotibiales por género a diferentes velocidades y lateralidad se reportan a continuación (tabla 1).

El promedio de torque en $\mathrm{Nm}$ para el género masculino a diferentes velocidades en forma bilateral para el cuádriceps fue el siguiente: a 60\% $/ \mathrm{seg}$ : $100.99 \pm 22.02 \mathrm{Nm}$, torque a 180\% $18 \mathrm{seg}: 77.48 \pm 15.43 \mathrm{Nm}$, torque a $300 \%$ seg: $68.56 \pm 14.14 \mathrm{Nm}$. Para el promedio de torque de isquiotibiales a diferentes velocidades de forma bilateral fue el siguiente: torque a $60 \% \mathrm{seg}$ : $69.33 \pm 15.24 \mathrm{Nm}$, torque a $180^{\circ} / \mathrm{seg}$ : $61.25 \pm 15.07 \mathrm{Nm}$, torque a $300^{\circ} / \mathrm{seg}$ : $61.15 \pm 16.50 \mathrm{Nm}$ (tabla 2).
El promedio de trabajo total expresado en Joules para los músculos cuádriceps de forma bilateral en hombres fue el siguiente: a $60^{\circ} / \mathrm{seg}$ : $478.14 \pm 126.36$ Joules, trabajo a $180^{\circ} / \mathrm{seg}: 738.97 \pm$ 205.40 Joules, trabajo a $300^{\circ} / \mathrm{seg}: 876.33$ \pm 232.88 Joules. Para el trabajo bilateral de isquiotibiales se obtuvo los siguientes promedios: trabajo a $60^{\circ} / \mathrm{seg}: 318.04 \pm$ 90.53 Joules, trabajo a 180\%/seg: 531.13 \pm 163.39 Joules, trabajo a $300^{\circ} / \mathrm{seg}$ : $659.59 \pm 179$. Joules (tabla 3).

El promedio de torque en $\mathrm{Nm}$ para el género femenino a diferentes velocidades de forma bilateral para cuádriceps fue el siguiente: torque a $60^{\circ} / \mathrm{seg}: 67.75 \pm 12.81$ $\mathrm{Nm}$, torque a $180^{\circ} / \mathrm{seg}: 50.35 \pm 9.11 \mathrm{Nm}$, torque a $300^{\circ} / \mathrm{seg}: 44.45 \pm 11.81 \mathrm{Nm}$. El promedio de torque de isquiotibiales a diferentes velocidades de forma bilateral fue el siguiente: torque a $60^{\circ} / \mathrm{seg}: 45.23 \pm 9.61$ $\mathrm{Nm}$, torque a $180^{\circ} / \mathrm{seg}: 38.15 \pm 8.69 \mathrm{Nm}$, torque a $300^{\circ} / \mathrm{seg}$ : $38.65 \pm 12.35 \mathrm{Nm}$ (tabla 4).

El promedio de trabajo total expresado en Joules para los músculos cuádriceps de forma bilateral en mujeres fue el siguiente: a 60\% $/ \mathrm{seg}$ : $337.58 \pm 98.56$ Joules, trabajo a 180\% seg: $476.99 \pm 92.41$ Joules, trabajo a $300^{\circ} / \mathrm{seg}$ : $564.33 \pm 106.18$ Joules. Para el trabajo bilateral de isquiotibiales se obtuvo los siguientes promedios: trabajo a 60\% $\mathrm{seg:} 223.44 \pm 70.52$ Joules, trabajo a 180\% $\mathrm{seg:} 323.21 \pm 83.20$ Joules, trabajo a 300\% seg: $415.99 \pm 75.78$ Joules (tabla 5).

En relación a la asociación de OAR y los promedios de valores de torque y traba- 
jo en hombres a velocidades de $60^{\circ} / \mathrm{seg}$, $180^{\circ} /$ seg y $300^{\circ} /$ seg no se encontró diferencia estadísticamente significativa en la prueba de ANOVA ( $p>0.05)$.

La asociación de OAR y los promedios de valores isocinéticos en mujeres se en- contró asociación significativa en la prueba de ANOVA ( $p<0.05$ ); en torque de cuádriceps izquierdo a $60^{\circ} / \mathrm{seg}$, torque de cuádriceps izquierdo a $180^{\circ} / \mathrm{seg}$, trabajo de cuádriceps derecho a $60 \% \mathrm{seg}$, trabajo de cuádriceps izquierdo a $60^{\circ} / \mathrm{seg}$.

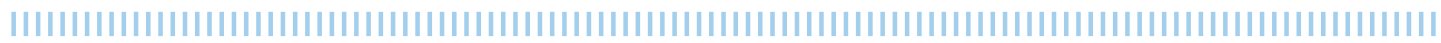

\section{Discusión}

Promedio de valores isocinéticos por género: El promedio de torque para cuádriceps por género en la muestra, se encuentra en rangos de normalidad para hombres a $60^{\circ} / \mathrm{seg}$ y a 180 y $300 \% \mathrm{seg}$. En mujeres se aprecia una relación de normalidad para velocidades a $180^{\circ} / \mathrm{seg}$ y $300^{\circ} / \mathrm{seg}$. Sin embargo a $60 \% \mathrm{seg}$, el promedio de torque para cuádriceps está por debajo de lo esperado en relación a valores de referencia tomados de Biodex Medical Systems (normative data base) que corresponden a una población adulta joven sin patologías, por lo que se puede explicar la diferencia de valores obtenidos, al tratarse de dos poblaciones diferentes. Esta variabilidad en los resultados fue estudiada por Yoon y colaboradores, quienes refieren que los valores isocinéticos pueden afectarse por diversos factores como la edad, género, posición durante la prueba, velocidad angular, efecto de la gravedad sobre el torque y características antropométricas. ${ }^{10}$

El promedio de torque de cuádriceps e isquiotibiales presentó un descenso inversamente proporcional al incremento de la velocidad de movimiento angular, lo cual se apreció en hombres y mujeres independiente de la dominancia. Yoon y colaboradores reportaron que el torque durante una prueba isocinética disminuye al incrementar la velocidad angular con o sin corrección de la gravedad. Esta disminución del torque ha sido atribuida a diferentes patrones de activación neurológica de unidades motoras a diferentes velocidades ${ }^{10}$, lo que se corrobora en el presente estudio.
La razón de equilibrio muscular de cuádriceps e isquiotibiales (C/I) presentó una disminución o empate en ambos géneros, al incrementar la velocidad angular de movimiento durante la valoración isocinética, lo que puede correlacionarse con mayor riesgo de lesiones musculares o ligamentarias producidas por desequilibrio muscular. Heewet reportó en una revisión sistemática que la corrección de la gravedad antes de la valoración isocinética muestra cambios significativos en la relación de cuádriceps/ isquiotibiales entre hombres y mujeres al incrementar la velocidad de movimiento angular. ${ }^{11}$ Este incremento en el torque de isquiotibiales se debe a un mecanismo reflejo que actúa con el fin de estabilizar y proteger el ligamento cruzado anterior a altas velocidades. ${ }^{12}$ Así pues los individuos con una razón $\mathrm{C} / \mathrm{I}<0.75$ presentan mayor riesgo de lesiones a altas velocidades.

En el género femenino se encontró diferencia en el promedio de torque de cuádriceps a $60^{\circ} / \mathrm{seg}$ al ser comparado con valores de normatividad para población adulta joven, sin embargo no se pudieron comparar los resultados de este estudio con valores de normatividad para trabajo y torque de isquiotibiales en ambos géneros. En un estudio realizado por Laura A. Wojick, al evaluar el torque de miembro pélvico, describió que existen diferencias entre hombres y mujeres, siendo más notables en periodos post-fatiga, además sugiere como probable causa, diferencias antropométricas, fisiológicas y de organización postural. ${ }^{13}$ 


\section{Promedio de valores isocinéticos en relación a lateralidad}

El análisis del promedio de valores isocinéticos en modo concéntrico/concéntrico de lado derecho con respecto al lado izquierdo, no presenta diferencia significativa en este estudio. Sin embargo, Saudi Abdulrahman $\mathrm{S}$. encontró que únicamente para el caso de la flexión, el torque en concéntrico es un $9.3 \%$ mayor de lado derecho (lateralidad dominante) comparado con el izquierdo, no encontrando diferencias en extensión. ${ }^{14}$

Promedio de valores isocinéticos en relación al IMC No se encontró asociación significativa entre las variables isocinéticas y el IMC en el presente estudio, a pesar de que más del $50 \%$ de la población estudiada padece de sobrepeso u obesidad. Algunos autores consideran que la obesidad es un factor de riesgo altamente asociado para osteoartrosis de rodilla y desequilibrios músculo esqueléticos ya que el exceso de peso puede contribuir a un incremento de carga mecánica y alterar la dinámica de movimiento. ${ }^{10,15}$ P. de vita, encontró que el torque de extensores de rodilla en los obesos es equivalente al del grupo de peso normal cuando caminan a la misma velocidad y un torque extensor menor en la rodilla en pacientes obesos cuando caminan a una velocidad seleccionada por ellos mismos, comparada con la velocidad estándar. ${ }^{11}$ Así, el torque demuestra la habilidad de algunos individuos obesos a reorganizar la función neuromuscular y a reducir la carga total de la articulación de rodilla. Este mecanismo de reorganización neuromuscular, puede ser una ventaja adaptativa que explique que aun con OAR y sobrepeso no encontremos asociación significativa en el torque de la rodilla de la muestra estudiada.

\section{Promedio de valores isocinéticos en relación a la edad}

El torque de cuádriceps a $60^{\circ} / \mathrm{seg}$ en mujeres fue menor al esperado según los rangos normales de pacientes adultos jóvenes. $B$. Danneskiold-Samsoe al medir los valores isocinéticos de rodilla, encontró que tanto en el hombre como en la mujer la fuerza muscular declina con la edad. Sosteniendo que el hombre es más fuerte que la mujer en todas las velocidades angulares. ${ }^{16}$ La disminución del torque en adultos mayores es atribuida a la pérdida progresiva de fibras musculares en número y tamaño, anormalidades en las propiedades contráctiles de las fibras musculares, alteraciones metabólicas, de excitación-contracción y patrones de activación neuromuscular alterados. ${ }^{17}$

\section{Promedio de valores isocinéticos en relación a OAR}

En el presente estudio se observó una asociación significativa en mujeres entre el torque en $\mathrm{Nm}$ y AOR de: cuádriceps izquierdo a $60^{\circ} / \mathrm{seg}$ (sig. 0.02); cuádriceps izquierdo a $180^{\circ} / \mathrm{seg}$ (sig.0.04); isquiotibial izquierdo a $180^{\circ} / \mathrm{seg}$ (sig.0.02); y en el trabajo en Joules de: cuádriceps derecho a $60^{\circ} / \mathrm{seg}$ (sig 0.02). Iwamoto reportó una reducción en el torque de cuádriceps en pacientes portadores de osteoartrosis de rodilla bilateral, causado por la disfunción articular y dolor. ${ }^{18,19}$

Las causas de disminución de torque en la OAR son multifactoriales. El dolor es la principal causa de inhibición en la activación muscular máxima en osteoartrosis al igual que el derrame articular, generando cambios patológicos en la marcha, aun en casos clínicamente sin relevancia. El torque en músculos de rodilla de pacientes con OAR puede presentar una reducción del $20 \%$ al $40 \%$ en comparación con pacientes sanos. ${ }^{9}$ 


\section{Conclusiones}

Los valores isocinéticos en adultos mayores de la UMFR Siglo XXI se encuentran en rangos normales tomando como referencia a pacientes jóvenes sanos, excepto en mujeres, donde se encontró asociación significativa de la OAR con disminución del torque y trabajo.

El promedio de torque de cuádriceps e isquiotibiales con respecto al género es mayor en hombres, sin presentar diferencia significativa en comparación con las mujeres. Con respecto el IMC y lateralidad no muestran asociación significativa, por lo que existen otros factores que influyen en el desarrollo de la OAR y no sólo el factor mecánico de sobrecarga de peso en rodillas, ya que este estudio sugiere que el desbalance muscular expresado mediante la relación $\mathrm{C} / \mathrm{I}$ y la disminución de torque y trabajo son factores para el desarrollo de la patología.

El grupo de mujeres que presentó asociación significativa de valores isocinéticos y OAR con una disminución en el torque de cuádriceps izquierdo a $60^{\circ} / \mathrm{seg}$ y $180 \%$ seg, torque de isquiotibial izquierdo a $180^{\circ} / \mathrm{seg}$ y trabajo de cuádriceps derecho a $60 \%$ seg, es probablemente causado por diferencias antropométricas, patrones de activación muscular y atrofia de fibras musculares relacionado con la edad, lo que condiciona una reducción de estos valores hasta en un $40 \%$ en relación a pacientes sanos. La OAR altera los patrones de activación muscular debido a dolor que puede desarrollarse durante la prueba y que se identifica fácilmente en los registros de torque y trabajo.

La velocidad de movimiento angular y el desequilibrio entre la relación de fuerzas de cuádriceps e isquiotibiales $(\mathrm{C} / \mathrm{I})$ es un factor de riesgo para producir lesiones ligamentarias y osteoraticulares que se han identificado en pacientes con OAR. El método de valoración isocinética permite identificar estos desbalances de forma objetiva, ofreciendo la posibilidad de dirigir el entrenamiento a grupos musculares en forma más controlada y preservar la función.

La valoración isocinética en adultos mayores es segura, pero requiere tiempo, altos costos y capacitación especial para poder llevarse a cabo, además de un adecuado juicio clínico para asegurarse de realizar en forma correcta dicha prueba.

La falta de estandarización de valores en adultos mayores y la variabilidad para realizar las pruebas de isocinecia muestran una desventaja para aplicar de forma rutinaria, por lo que se sugiere estandarizar una prueba para incrementar la evidencia.

Este tipo de valoraciones ofrece la ventaja de dar resultados objetivos en cuanto a parámetros de torque y trabajo muscular, identificando desbalances musculares dependiendo del grupo muscular evaluado, además permite al médico de rehabilitación y fisioterapeutas dirigir un entrenamiento específico a éstos.

Nota: Este trabajo fue presentado en extenso ante el consejo Mexicano de Medicina de Rehabilitación en 2014 y obtuvo el primer lugar como trabajo de tesis para egresados de la especialidad. 


\section{Bibliografía}

1. Altman $R$, Asch $E$, Bloch $D$, Bole $G$, Borenstein $D$, Brandt K, et al. Development of criteria for the classification and reporting of osteoarthritis: classification of osteoarthritis of the knee. Arthrit Rheum.1986; 29:1039-49.

2. Espinosa Morales R, Arce Salinas A, Cajigas Melgoza J C, Esquivel Valerio J A, Gutiérrez Gómez J J, Martínez Hernández $J \mathrm{~L}$, et al. Reunión multidisciplinaria de expertos en diagnóstico y tratamiento de pacientes con Osteoartrosis. Med Int Mex. 2013; 29(1) 67. 92.

3. OMS.org [Internet]. Organización Mundial de la Salud. Actualizado el 12 de Noviembre de 2013; consultado el 15 de Nov de 2013 .Disponible en: http:// www.who.int/topics/ageing/es/

4. Motivos de demanda de consulta externa (DITIES 2007). Unidad de investigación, educación y políticas en Salud, División técnica de información y estadística en Salud. México: División de prestaciones médicas.

5. Tan J, Balci N,Sepici V, Gener FA. Isokinetic and isometric strength in osteoarthrosis of knee. A comparative study with healthy women. Am J Phys Med Rehab.1995; 74(5) 364-369.

6. Terreri $A$, .Ambrosio $M A$, Pedrinelli A, Albuquerque $R$, Andrusaitis $F$, Greve J $M$, et al. Isokinetic assessment of the flexor-extensor balance of the knee in athletes with total rupture of the anterior cruciate ligament. Hosp Clin Fac Med Sao Paulo.1999; 54(2) 35-8.

7. Crayton $L M$, Wright $T$. Comparison of three methods of assessing muscle strength and imbalance ratios of the knee. J Athel train .1993; 28(1):1-4.

8. Devan $M R$, Pescatello $L S$, Faghri $P$, Anderson J. A prospective study of overuse knee injuries among female athletes with muscle imbalances and structural abnormalities. J Athel train. 2004;39(3):263-267.

9. Palmieri-Smith RM, Thomas AC, Karvonen-Gutierrez C. Isometric quadriceps strength in women with mild, moderate and severe knee osteoarthritis. Am J Phys Med Rehabil.2010;89(7): 541-548.

10. Yoon T S, Park D S, Kang SW, Chun S, Shin JS. Isometric and isokinetic torque curves at the knee joint. Younsei Medic Journal.1991; 32 (1) 33-42.

11. Vita DP, Hortobaqyi T. Obesity is not associated with increased knee joint torque and power during level walking. J of Biomech.2003;36 (9)1355-1362.

12. Timothy E.H, Myer GD, Zazulak B T. Hamstrings to quadriceps peak torque ratios diverges between sexes with increasing isokinetic angular velocity. J of Sci and Med in Sport .2008;11(5) 452-459.

13. Wojcik L A., Maury A, Dinging Lin, Shibata P A. Madigan $M L$. Age and gender moderate the effects of localized muscle fatigue on lower extremity joint torques used during quiet stance. Hum Mov Sci. 2011; 30(3) $574-583$

14. Abdulrahman $\mathrm{S}$, Alangari $\mathrm{H} M$. Normal isometric and isokinetic peak torques of hamstring and quadriceps muscles in young adult Saudi males. J Neurosci.2004; 165-170.

15. Graeme T. Harding C L, Dunbar M J, Stanish W D, Astephen Wilson J L. Body mass index affects knee joints mechanics during gait with and without moderate knee ostearthritis. Osteoarthritis Cartilage.2012 20(11)1234-1242.

16. Danneskiold-Samsoe $B$, Bartels $E M$, Bülow $P M$, Stockmarr A, Holm C C. et al. Isokinetic and isometric muscle strength in a healthy population with special reference to age and gender. Acta Physiol.2009; 197(673):1-68.

17. Clark DJ, Fielding RA. Neuromuscular contributions to age-related weakness. J Gerontol A Biol Sci Med Sci 2012; 67(1):41-7.

18. Iwamoto J, Takeda T, Sato Y. Effect of muscle strengthening exercises on the muscle strength in patients with osteoarthritis of the knee. The Knee. 2007;14 :224-230

19. O'Reilly S C, Jones A, Muir K R, Doherty M. Quadriceps weakness in knee osteoarthritis: the effect on pain and disability. Ann Rheum Dis.1998;57:588-94. 


\section{Tabla I}

Promedio de valores isocinéticos por lateralidad y género a diferentes velocidades (lado derecho/ lado izquierdo) en la población adulta mayor de la UMFR siglo XXI

\begin{tabular}{|lcccc|}
\hline Músculos a diferentes velocidades & \multicolumn{2}{c}{ MASCULINO } & \multicolumn{2}{c|}{ FEMENINO } \\
\hline & Derecho & Izquierdo & Derecho & Izquierdo \\
\hline TC $60^{\circ} / \mathrm{seg}$ & 103.6 & 98.4 & 66.8 & 68.7 \\
\hline TC $180^{\circ} / \mathrm{seg}$ & 78.2 & 76.8 & 50.6 & 50.1 \\
\hline TC $300^{\circ} / \mathrm{seg}$ & 68.5 & 68.7 & 45.6 & 43.3 \\
\hline TI $60^{\circ} / \mathrm{seg}$ & 71.0 & 67.7 & 45.8 & 44.7 \\
\hline TI $180^{\circ} / \mathrm{seg}$ & 63.8 & 58.7 & 39.0 & 37.3 \\
\hline TI $300^{\circ} / \mathrm{seg}$ & 62.1 & 60.2 & 39.0 & 38.3 \\
\hline TRAB C $60^{\circ} / \mathrm{seg}$ & 493.8 & 462.4 & 344.3 & 330.9 \\
\hline TRAB C $180^{\circ} / \mathrm{seg}$ & 755.0 & 722.9 & 488.5 & 465.5 \\
\hline TRAB C $300^{\circ} / \mathrm{seg}$ & 882.4 & 870.2 & 563.2 & 565.4 \\
\hline TRAB I $60^{\circ} / \mathrm{seg}$ & 320.7 & 315.4 & 230.0 & 216.9 \\
\hline TRAB I $180^{\circ} / \mathrm{seg}$ & 539.8 & 522.5 & 314.7 & 331.7 \\
\hline TRAB I $300^{\circ} / \mathrm{seg}$ & 659.6 & 653.6 & 410.1 & 421.8 \\
\hline
\end{tabular}

Fuente: UMFRSXXI Torque en Nm, trabajo en Joules.

TC: Torque de cuádriceps.

TI: Torque de isquiotibiales.

TRAB C: Trabajo de cuádriceps.

TRAB I: Trabajo de isquiotibiales.

\section{Tabla 2}

Torque de cuádriceps e isquiotibiales bilateral a diferentes velocidades en hombres adultos mayores de la UMFR Siglo XXI

\begin{tabular}{|lcccccc|}
\hline & \multicolumn{3}{c}{ Cuádriceps } & \multicolumn{3}{c|}{ Isquiotibiales } \\
\hline & $60^{\circ} / \mathrm{seg}$ & $180^{\circ} / \mathrm{seg}$ & $300^{\circ} / \mathrm{seg}$ & $60^{\circ} / \mathrm{seg}$ & $180^{\circ} / \mathrm{seg}$ & $300^{\circ} / \mathrm{seg}$ \\
\hline Promedio & 100.99 & 77.48 & 68.56 & 69.33 & 61.25 & 61.15 \\
\hline Desviación típica & 22.02 & 15.43 & 14.14 & 15.24 & 15.07 & 16.50 \\
\hline Mínimo & 42.30 & 50.50 & 44.50 & 46.75 & 40.35 & 42.30 \\
\hline Máximo & 149.95 & 108.55 & 105.90 & 98.65 & 98.15 & 105.80 \\
\hline
\end{tabular}

Torque en $\mathrm{Nm}$

Fuente: UMFR SXXI

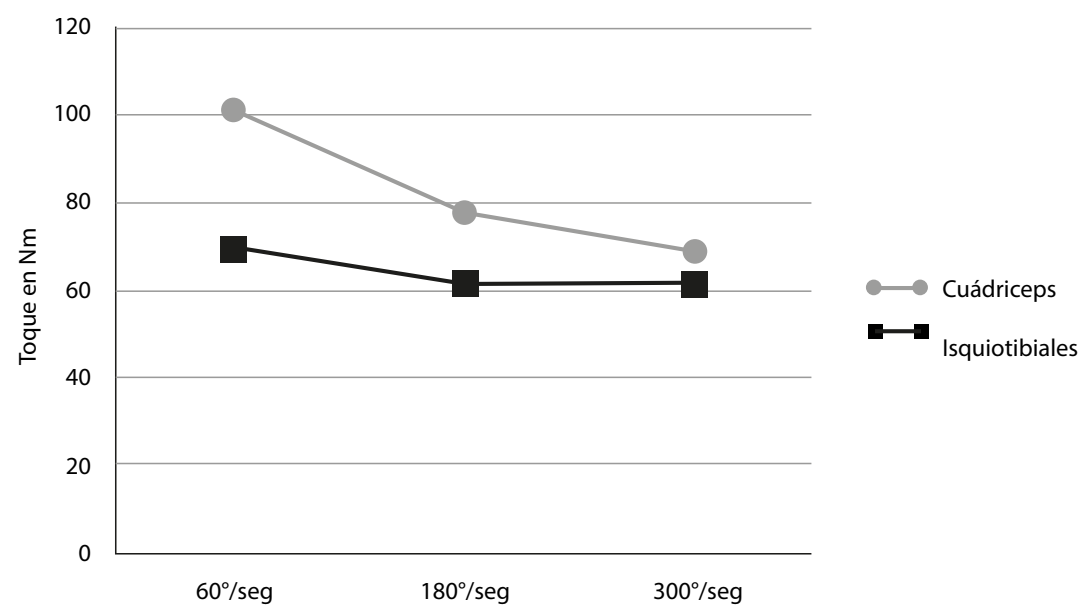




\section{Tabla 3}

Trabajo de cuádriceps e isquiotibiales bilateral a diferentes velocidades en hombres adultos mayores de la UMFR siglo XXI

\begin{tabular}{|lcccccc|}
\hline & \multicolumn{3}{c}{ Cuádriceps } & \multicolumn{3}{c|}{ Isquiotibiales } \\
\hline & $60 \%$ seg & $180^{\circ} / \mathrm{seg}$ & $300^{\circ} / \mathrm{seg}$ & $60^{\circ} / \mathrm{seg}$ & $180^{\circ} / \mathrm{seg}$ & $300^{\circ} / \mathrm{seg}$ \\
\hline Promedio & 478.14 & 738.97 & 876.33 & 318.04 & 531.13 & 656.59 \\
\hline Desviación típica & 126.36 & 205.40 & 232.88 & 90.53 & 163.39 & 179.10 \\
\hline Mínimo & 211.95 & 282.70 & 386.95 & 184.95 & 293.35 & 379.00 \\
\hline Máximo & 838.00 & 1232.00 & 1290.95 & 575.85 & 928.45 & 1026.35 \\
\hline
\end{tabular}

Trabajo en Joules.

Fuente: UMFRSXXI

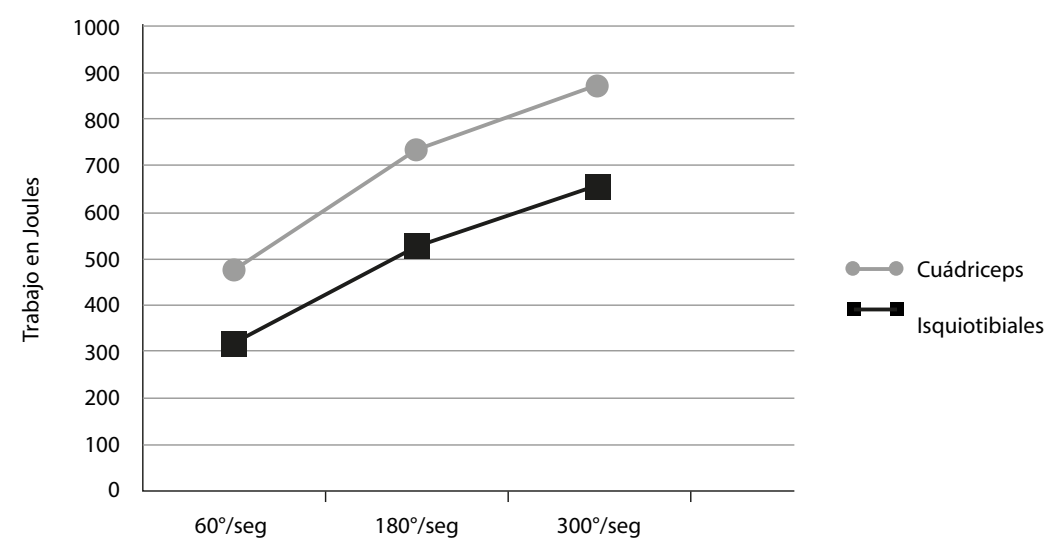

\section{Tabla 4}

Torque de cuádriceps e isquiotibiales bilateral a diferentes velocidades en mujeres adultas mayores de la UMFR Siglo XXI

\begin{tabular}{|lcccccc|}
\hline & \multicolumn{3}{c}{ Cuádriceps } & \multicolumn{3}{c|}{ Isquiotibiales } \\
\hline & $60^{\circ} / \mathrm{seg}$ & $180^{\circ} / \mathrm{seg}$ & $300^{\circ} / \mathrm{seg}$ & $60^{\circ} / \mathrm{seg}$ & $180^{\circ} / \mathrm{seg}$ & $300^{\circ} / \mathrm{seg}$ \\
\hline Promedio & 67.75 & 50.35 & 44.45 & 45.23 & 38.15 & 38.65 \\
\hline Desviación típica & 12.81 & 9.11 & 11.81 & 9.61 & 8.69 & 12.35 \\
\hline Mínimo & 47.20 & 35.90 & 29.00 & 27.15 & 25.05 & 21.25 \\
\hline Máximo & 94.60 & 67.40 & 72.60 & 60.65 & 60.40 & 68.95 \\
\hline
\end{tabular}

Torque en $\mathrm{Nm}$

Fuente: UMFR SXXI

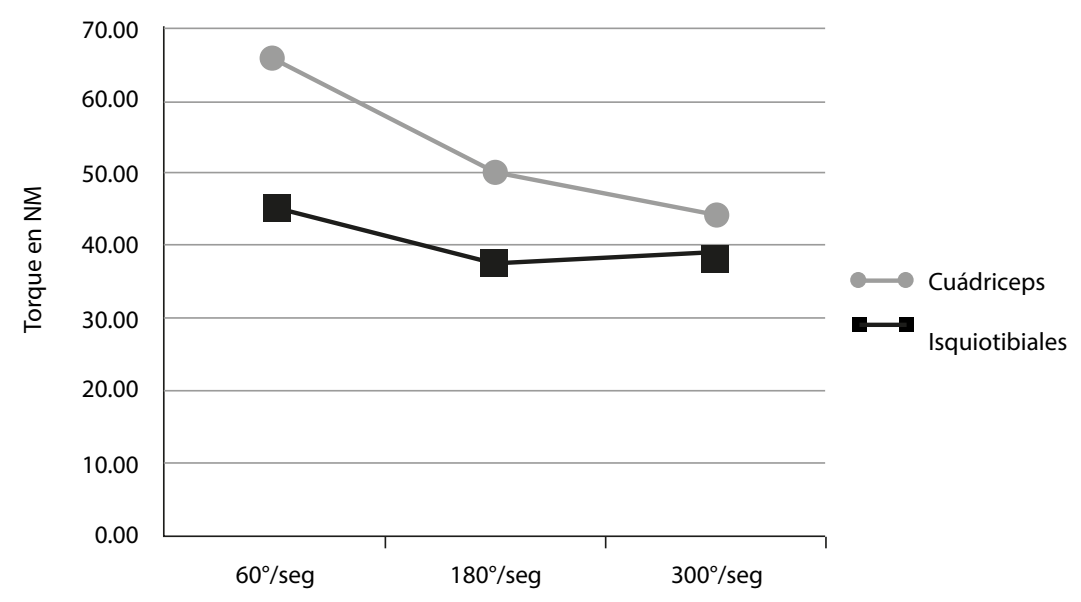




\section{Tabla 5}

Trabajo de cuádriceps e isquiotibiales bilateral a diferentes velocidades en mujeres adultas mayores de la UMFR Siglo XXI

\begin{tabular}{|c|c|c|c|c|c|c|}
\hline \multirow[b]{2}{*}{$60^{\circ} / \mathrm{seg}$} & \multicolumn{3}{|c|}{ Cuádriceps } & \multicolumn{3}{|c|}{ Isquiotibiales } \\
\hline & $180^{\circ} / \mathrm{seg}$ & $300^{\circ} / \mathrm{seg}$ & $60^{\circ} / \mathrm{seg}$ & $180^{\circ} / \mathrm{seg}$ & $300^{\circ} / \mathrm{seg}$ & \\
\hline Promedio & 337.58 & 476.99 & 564.33 & 223.44 & 323.21 & 415.99 \\
\hline Desviación típica & 98.56 & 92.41 & 106.18 & 70.52 & 83.20 & 75.78 \\
\hline Mínimo & 213.40 & 341.50 & 390.45 & 114.60 & 184.85 & 278.55 \\
\hline Máximo & 654.55 & 652.35 & 771.75 & 440.15 & 562.60 & 546.00 \\
\hline
\end{tabular}

Trabajo en Joules.

Fuente: UMFR SXXI

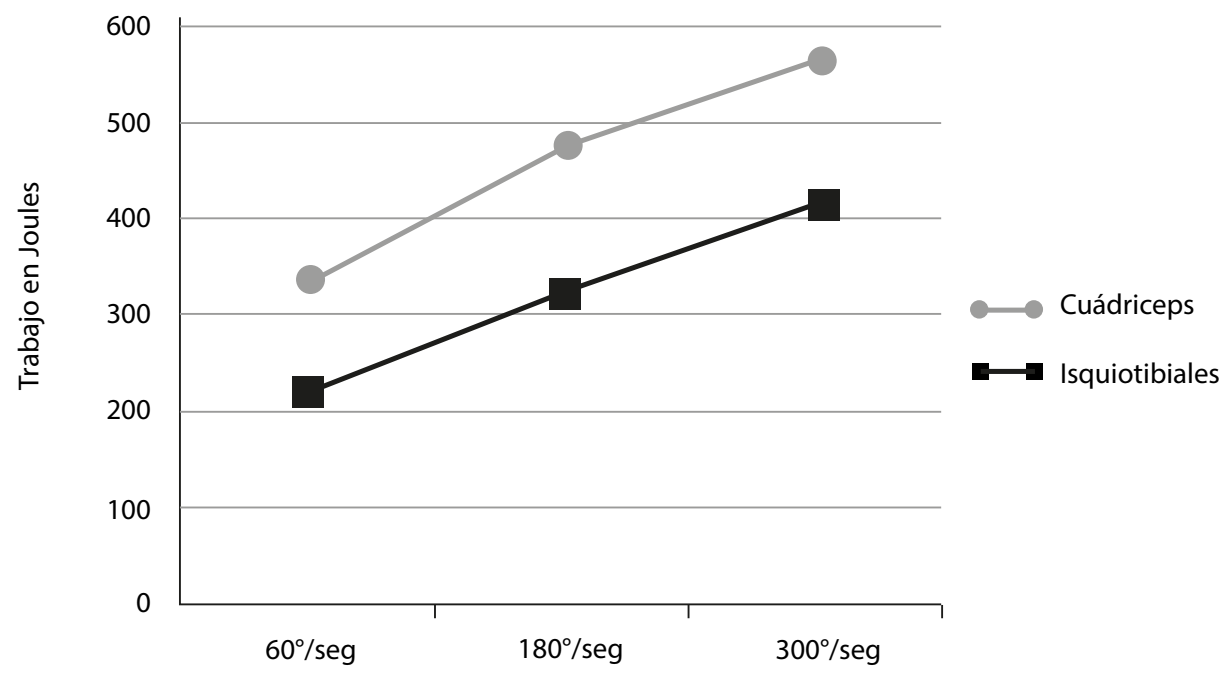

